\title{
Medical and Economic Impact of the Gluten-Free Diet on the Quality of Life and Social Adaptation in Patients with Gluten Enteropathy - a Ten-Year Clinical Experience
}

\author{
Assist. Prof. Lili Grudeva, MD, PhD \\ Clinic of Gastroenterology, St. Marina University Hospital-Varna, Medical University of Varna \\ grudeva63@abv.bg
}

\begin{abstract}
The gluten-free diet (GFD) is complex, costly and influences all activities related to nutrition, which makes it hard to follow throughout the entire life. The avoidance of a wide spectrum of foods containing gluten often requires significant changes in the dietary habits and the lifestyle.
\end{abstract}

Keywords: gluten enteropathy, gluten-free diet, quality of life, social adaptation, emotional impact

JEL Code: I120; doi:10.36997/IJUSV-ESS/2019.8.3.163

\section{Introduction}

Many factors may influence the desire and ability to change already established dietary habits. Therefore, there is a need for better understanding of the emotional impact of the gluten-free diet (GFD) on the overall quality of life and the difficulties resulting from it.

\section{Aim}

The aim of the study is to assess the emotional impact, the food-related difficulties and strategies used in everyday situations, which are a consequence of GFD. The presented results encompass the dietetic aspects of the disease.

\section{Materials and Methods}

The clinical study includes 112 patients with gluten enteropathy (GE) and 22 with gluten sensitivity, used as a control group. It took place in the period from January 2005 to June 2015.

The questionnaire contains 59 questions divided by demographic data, diagnosis, symptoms, diet adherence, information sources, GFD knowledge, emotional impact, difficulties experienced and the strategies used during the diet.

The questions regarding the usefulness of the information sources, the emotional impact, the difficulties and strategies use a five-point scale with the following options: never, rarely, sometimes, often, and very often.

The data is analyzed with the help of the statistical functions of Microsoft Excel. The results are presented as percentages. All investigated patients have undergone a small bowel biopsy with a confirmed diagnosis. The results reported in the current study are based on these biopsies.

\section{Results and Discussion}

Out of all patients included in our study, 2 are only with $8^{\text {th }}$ grade education, 28 are with secondary education, 6 have college diploma and 22 have graduated from a university.

\section{Frequency of Intentional Gluten Consumption}

The majority of the surveyed $(66 \%)$ indicate that they have never consumed gluten intentionally. A total of $17 \%$ say that they have intentionally strayed from the diet consuming gluten 1-2 times in the previous year. The remaining 17\% report intentional gluten consumption at least once a month within the previous year. The percentage of respondents who have intentionally consumed gluten at least once in the previous year is lower among those who have followed GFD for a longer period of time $(p=0.013)$. There is no significant difference in the frequency of 
intentional consumption among men and women.

\section{Reasons for Avoiding Gluten Consumption}

The majority of the surveyed $(87.8 \%)$ indicate that they consider the prevention of long-term complications and the avoidance of adverse reactions equally important reasons to avoid gluten consumption. In addition to these data, 9.9\% report the long-term complications to be more important than the adverse reaction, and $2.2 \%$ think that adverse reactions are an important reason to avoid gluten consumption.

\section{GFD Information}

Patients use different sources for information on GFD.

Table 1. Information sources and their usefulness assessed as good/very good

\begin{tabular}{|l|c|c|}
\hline \multicolumn{1}{|c|}{ Information Source } & $\begin{array}{c}\text { Percentage of Patients Assessing } \\
\text { It as Good/Very Good }\end{array}$ & Number \\
\hline 1. Internet & $53.2 \%$ & 30 \\
\hline 2. Dietitian & $72 \%$ & 58 \\
\hline 3. Medical books/journals & $12 \%$ & 32 \\
\hline 4. Other people with GE & $51 \%$ & 58 \\
\hline 5. Gastroenterologist & $43.9 \%$ & 54 \\
\hline 6. General practitioner & $20.6 \%$ & 52 \\
\hline 7. Alternative medicine & $3.6 \%$ & 58 \\
\hline
\end{tabular}

\section{Usefulness of GFD Information}

The patients' view of the information source varies, from $72 \%$ for the nutrition specialist to $20.6 \%$ for the general practitioner.

The percentage of surveyed individuals in terms of recognition of allowed and forbidden foods increases with the increase of the period of adherence to the diet - from $38 \%$ for those being on GFD for less than a year since diagnosis, to $45 \%$ in patients following the diet for a period between 1 and 5 years, and reaching 52.2\% when adhering to the diet for more than 5 years.

\section{Emotions Related to GFD}

The participants are asked to report their emotions before the diagnosis and in the first months after being diagnosed. The majority report that they accept the diet and feel relieved that their diagnosis has been established at last. Women are quicker to adapt in comparison to men with a statistically significant difference (L. Grudeva, 2018).

There is a significant difference between men and women during the month before the diagnosis and the first months on GFD, $\mathrm{p}<0.01$.

The negative emotions, which are often reported, are considerably more in the months after the diagnosis than before that. Women report more negative emotions for both periods.

Twelve out of 39 states, which are a burden for the patients, are reported in a higher percentage of women than men. The remaining 27 are equally difficult for both sexes.

Those, who have followed the diet for 5 or more years, experience fewer difficulties. Nevertheless, more than half of the participants experience difficulties in $1 / 3$ of the situations they encounter. They include a limited choice of products and dishes when visiting a restaurant, café or fast food venues. In addition, their fears stem from the fact that manufacturers are not always honest enough when labeling gluten-free products, and last but not least is the higher price compared to their gluten equivalents. They are also afraid that in most cases the chefs in restaurants are insufficiently trained in preparing gluten-free dishes, and that there is a limited food choice outside their home and especially when traveling. A very high percentage, more than a half, express 
disappointment that their relatives and friends feel sorry for them. A very small percentage blame their families for the lack of understanding and sympathy. A total of $24.3 \%$ indicate that they suspect that their relatives and friends are afraid to invite them for a visit.

Table 2. Emotions, which patients on GFD often/very often experience

\begin{tabular}{|l|c|c|c|c|c|c|}
\hline $\begin{array}{c}\text { Emotions } \\
\text { Often/Very Often }\end{array}$ & \multicolumn{3}{|c|}{$\begin{array}{c}\text { During the First Months } \\
\text { After Diagnosis (\%) }\end{array}$} & \multicolumn{3}{c|}{$\begin{array}{c}\text { During the Month Before } \\
\text { Diagnosis (\%) }\end{array}$} \\
\hline & All & Men & Women & All & Men & Women \\
\hline Positive emotions & & & & & & \\
\hline Relief & 57 & 61 & 55 & 42.6 & 40.8 & 43 \\
\hline Acceptance & 56 & 63 & 51 & 62.9 & 59.4 & 66.3 \\
\hline Negative emotions & & & & & & \\
\hline Disappointment & 56.9 & 47 & 60 & 20.8 & 16 & 20.5 \\
\hline Overwhelmed & 47.7 & 35 & 49 & 7.7 & 4.5 & 7 \\
\hline Isolated & 42.7 & 29.7 & 43 & 17.6 & 10.6 & 21 \\
\hline Confused & 36.4 & 30.7 & 45.8 & 5.1 & 5 & 5.3 \\
\hline Anxious & 36 & 25.9 & 41 & 8.8 & 7.9 & 8.1 \\
\hline Sad & 33.8 & 22.8 & 43 & 8.3 & 4.8 & 7.9 \\
\hline Angry & 30.2 & 19.8 & 33.8 & 8.3 & 6.1 & 8.2 \\
\hline Depressed & 24.5 & 14.3 & 27 & 6.7 & 4.3 & 12 \\
\hline
\end{tabular}

Other specific difficulties come from the confusion about the intake of different medicaments and vitamins.

A significant part of the patients feels guilty about the possibility of their children inheriting their condition $-24.6 \%$.

The questions that the patients are asked are grouped into several categories - situations that they encounter and must deal with:

- Buying gluten-free food

- Food preparation

- Eating with family and friends

- Eating out (restaurant, fast food venues)

- Eating when traveling

- Eating at work

The highest number of patients replied to 17 of the questions.

Between 0 and 4 strategies are used by $18 \%$ of the questioned.

Between 5 and 7 are used by $32.1 \%$.

Between 8 and 10 are used by $29 \%$ of the surveyed.

$2.2 \%$.

Between 11 and 13 strategies are used by $15 \%$, and more than 14 strategies are used by

Fig. 1 presents the results on the percentage of respondents indicating a total of 5 levels of strategies used, grouped into 3 categories of intentional consumption of gluten-containing foods: „never consume intentionally“, "intentionally consume 1 or 2 times per year", and ,intentionally consume gluten-containing food at least once in the past year".

The figure shows that the respondents, who most frequently "sin" are the ones using the lowest number of strategies: between 0 and 4 (34\%), those consuming 1-2 times per year are 17\%, and those who never consume are $66 \%$. In general, those using more strategies are considerably less likely to intentionally consume gluten $(\mathrm{p}<0.001)$.

This correlation is observed among both men and women, although on average men use 
fewer strategies than women.

\section{Study on the Strategies for Intentional Consumption of Gluten-Containing Products}

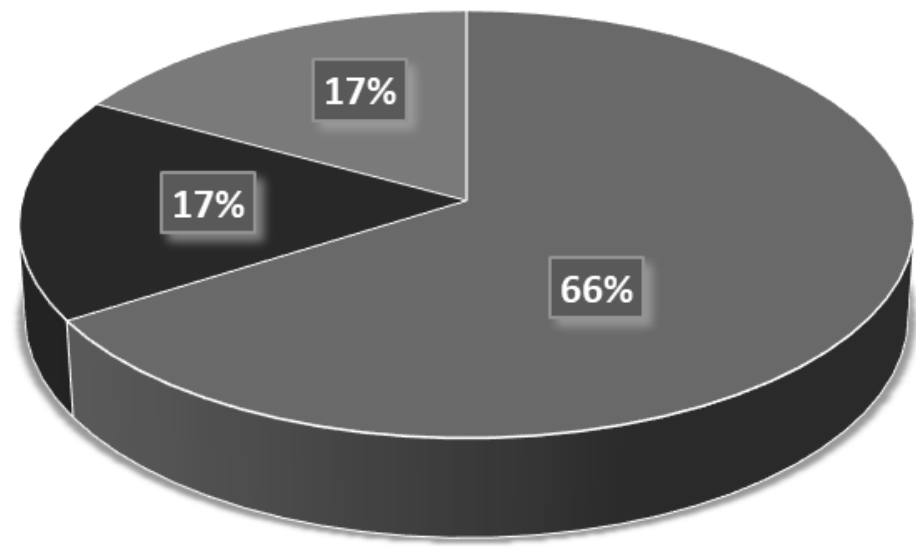

- never consume

intentionally

- intentionally consume 1 or 2 times

- intentionally consume at least once in the past year

Fig. 1. Study on the strategies for intentional consumption of gluten-containing products

Many factors are related to the change in the models of health behavior, including personal ones, cultural influence, social relationships. A large part of the patients perceives GFD as a burden even after following the diet for several years.

\section{Information and Knowledge About GFD}

It is obvious that the understanding of the diet and the learning of skills on dealing with different situations can alleviate the burden and help with the adherence to GFD (McKenzie et al., 2010). The awareness about the condition must be increased, especially among general practitioners and dietitians, in order to improve patient care and help with the decrease of the social, personal and medical expenditure related to the disorder.

Women show better awareness about GFD compared to men. The longer period of diet adherence logically leads to better results in regard to the knowledge about allowed and forbidden foods.

When it comes to the emotional impact of GFD, the current study shows that there is a need for changes in the eating habits throughout the entire life, which leads to a significant emotional impact. It confirms the fact that women undergo a worse emotional stress than men, which corresponds with the data from Harlett et al., 2003. The role of women in all aspects of the diet, at home and outside it, and the inherent gender differences are among the other possible factors.

The most frequent emotion reported equally by men and women is relief after the diagnosis. After many years of an unexplained ailment, a significant proportion of the patients report a great 
relief, that their do not suffer an incurable disease, but a condition treatable only with a diet. However, soon after starting the new nutritional regimen, the sense of relief decreases sharply.

The patients report feeling disappointed, overwhelmed and isolated, often they are anxious, sad or angry. Frustration and isolation are among the most frequent negative emotions, even among those following the diet for more than 5 years. It is important for this emotion to be shared so that people can overcome this difficulty.

The acceptance of the condition and its treatment include a process of adaptation to the new conditions, and time to learn to live with these changes. In the current study the percentage of men accepting the new diet is higher in the beginning, but with the passing of time women demonstrate better adaptation to the diet.

\section{Difficulties and Experience in Diet Adherence}

The current research allows us to assess the quantity, frequency and variety of difficulties and strategies used, as well as the different behavior in men and women, and the impact of GFD on their life for different periods of time.

Women experience approximately $1 / 3$ of the identified difficulties, significantly more often than men. The experience after longer adherence to the diet influences the frequency and tolerance of the difficulties related to buying and preparing of food, and its delivery from outside places. The facts demonstrate a lower percentage of difficulties when GFD is followed for 5 or more years, similar to the data from Lee et al., 2012. There is a significant decrease in the negative impact of the diet with the passing of time, although there are still some problematic situations, like dining out or when traveling.

More than $3 / 4$ of the studied patients, even those who have followed the diet for 5 or more years, continue to experience difficulties related to food labeling and honest assessment of the gluten-free ingredients in the content, a limited choice of restaurants and other places for eating out, cafes, etc. This is reported by other studies, leading to preliminary planning, prioritization of problems and their solution.

The high price of prepackaged gluten-free products is identified as a limiting factor by more than $70 \%$ of the respondents. The need for additional cooking time is defined as a problem by the majority of patients. The preparation of food and eating at home are the cheaper alternative, but this requires additional skills. We hope that the market competition will decrease the price differences between GF products and their gluten equivalents.

More than half of the patients experience a feeling of self-pity and shame that they have different nutritional needs and this must be discussed during nutritional consultations. The positive attitude to the food and its health benefits should be encouraged.

The support of the family and friends is very important for those on GFD. The results from the survey are uplifting. Only 2.9\% do not encounter the support of their families, and 5.3\% - of their friends. Almost $1 / 4$ suspect that this diet can be a cause for their isolation.

The statistically significant reverse correlation between the strategy used and the intentional consumption of gluten is of considerable importance for the people managing the disease. The basic difficulties experienced by more than half of the studied patients are related to the limited choice of food outside the home and the worry about the guaranteed safe eating. Therefore, more efforts are needed to guarantee a higher quality service in food venues and the overall training of those working in this sphere.

\section{Quality of Life (QoL) and Coping Strategies}

The problem with QoL and its improvement at the university, the workplace, the restaurant and the phrase "gluten-free diet", very often do not require further clarification, they must be understood! 
A review of the literature over time shows that QoL of GE patients before and after diagnosis is strongly influenced by the presence or lack of gastrointestinal symptoms. QoL of GE patients before the diagnosis and after its establishment is mainly related to the difficulties of a chronic condition and the limitations of GFD and its proper adherence. Improper diet adherence is linked to lower QoL, but it is hard to determine the main reason for this - the cause or the GFD effect.

The long duration of the symptoms before the diagnosis - gastrointestinal, neurological, psychiatric and concomitant symptoms, is a predictor of decreased QoL.

In the recent years there has been a considerable increase in the awareness about this disorder, both among physicians and among the general public. The patients with gastrointestinal problems are still a vulnerable group when it comes to the adaptation to this chronic condition, especially in terms of their social life. The planned intervention in the diagnosis and follow-up will indisputably improve the adaptation to this condition and its limitations.

\section{QoL Assessment of GE Patients}

For GE patients GFD adherence might be restrictive, hard to follow and it may negatively influence their QoL. Despite the fact that in recent years, gluten-free products have become available and there are new ordinances on allergens and labeling within the European Union, the exclusion of gluten-containing foods is hampered by the confusing labeling of products, the limited availability and the high price of gluten-free alternatives. In almost all identified studies there is a tendency for a higher percentage of non-adherence to GFD among adults diagnosed as children than among those diagnosed in adulthood. In our case a very small percentage of the patients have known about their condition since childhood, which provides us with ground to draw statistically significant conclusions. Our observations are in accordance with those of two major studies of a group of Italian adult patients, where a correlation between the adherence, the level of education, and the socio-economic status was established.

All age groups report significantly increased QoL during the study compared to the period before the diagnosis.

In this study we have established that QoL of GE patients before the diagnosis significantly differs from that of the general population. There is a difference of 0.56 to 0.84 similar to the QoL of stroke patients.

The advantage of FQ-5D is that it is a widely used generic QoL instrument, which allows comparison.

Our observations show that the participants with more severe malabsorption symptoms are more likely to adhere strictly to the diet and are more motivated.

Regular diet follow-up and disease discussion with the doctor are related to better diet adherence.

The membership of patients from Western societies in support groups shows considerably higher levels of diet adherence even though this information is not always known and it is difficult to draw definitive conclusions about the impact of this membership. Unfortunately, the lack of such communities in Bulgaria does not give us ground to conclude anything in this regard.

The complete non-adherence to GFD is unusual but according to our data, partial nonadherence is observed in more than $50 \%$ of the participants, which is comparable with literature data. There are numerous demographic, psychosocial and cultural factors related to moving to GFD. Those most strongly related to diet adherence are information, emotional, socio-cultural factors and regular diet follow-up. However, the proof in support of these conclusions is limited. There is a weak correlation between diet adherence and practical difficulties related to the diet complexity, accessibility of gluten-free alternatives and educational level. 


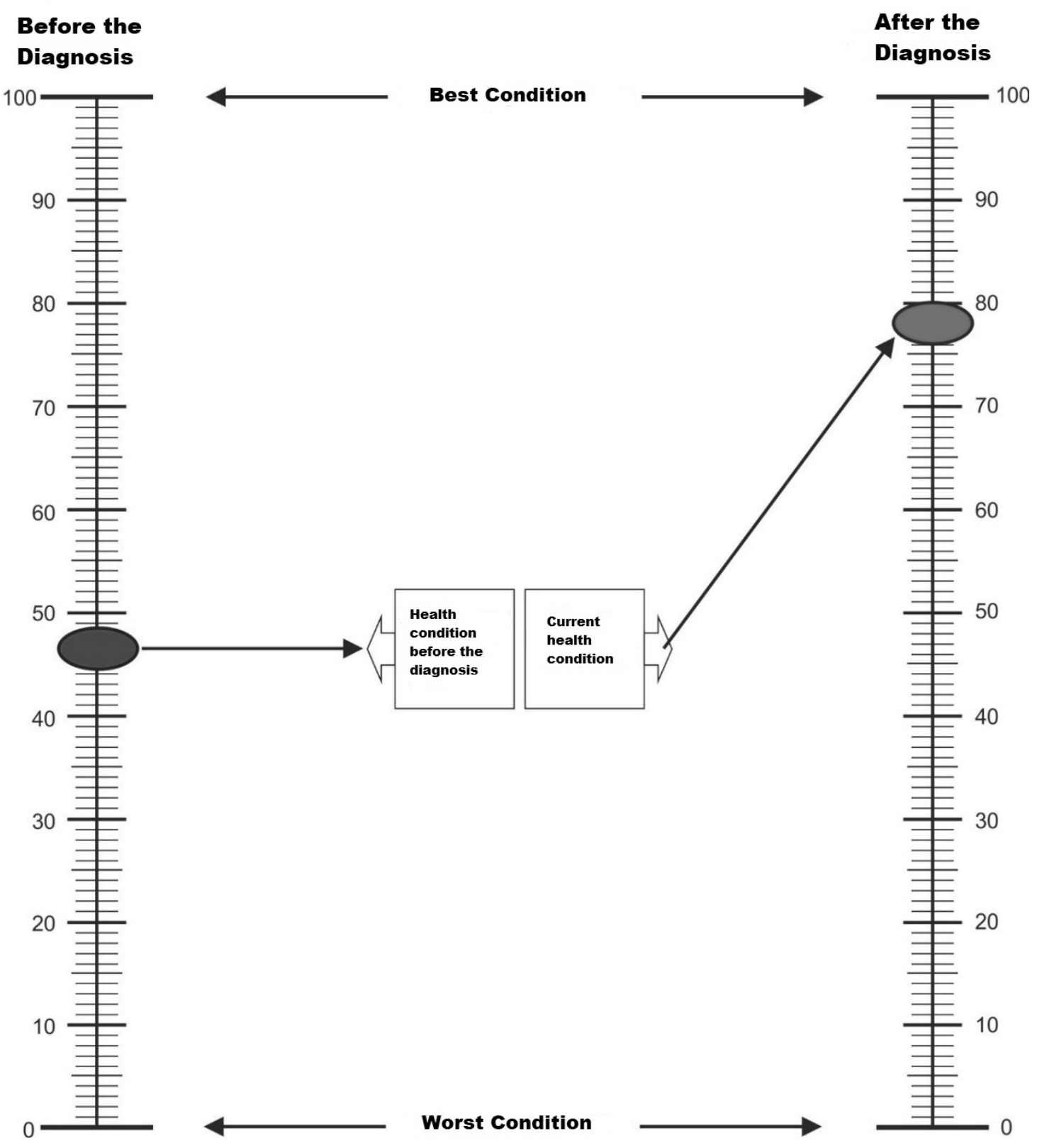

Fig. 2. Visual analog scale of QoL self-assessment before and after the diagnosis

The main limitation of our conclusions is the lack of comparability between studies in terms of design, methodology, definitions and diet adherence measurement. There is no gold standard for measurement and monitoring of diet adherence. Histological changes may take more than 12 months to be reversed after starting GFD and the repeated biopsies are not always an acceptable option for monitoring. Serologic tests are not sensitive enough to discover "small dietary transgressions" and their link to the histological results are disputable. The assessment of an expert dietitian in the field of GE is based on an interview or a nutritional diary, and is considered the most objective non-invasive method of measuring diet adherence. Moreover, the lack of a unanimous expert opinion on what strict GFD consists of is an important clinical problem because the concept of a safe threshold of gluten consumption and the variations in the individual tolerability is still a point of debate. 


\section{Conclusion}

This article presents an attempt to systematically identify the factors influencing GFD adherence in adult patients. It does not examine the effect of the different levels of diet adherence and cannot provide definitive conclusions about the patients' long-term clinical results. It is possible that in the future the conditions under which the patients stray from GFD may become known, and they will help with the development of suitable and effective strategies to help those who need support during their treatment.

\section{References}

1. Zarkadas, M., Cranney, A., Case, S., Molloy, M., Switzer, C, Graham, I., et al. (2006) The impact of a gluten-Free diet on adults with coeliac disease: Results of a national survey. Journal of Human Nutrition and Dietetics. 19(1). pp. 41-49.

2. Zarkadas, M., Dubois, S., MacIsaac, K., Cantin, I., Rashid, M., Roberts, K., et al. (2013) Living with coeliac disease and a gluten-Free diet: A canadian perspective. Journal of Human Nutrition and Dietetics. 26(1). pp. 10-23.

3. MacCulloch, K. and Rashid, M. (2014) Factors affecting adherence to a gluten-free diet in children with celiac disease. Paediatrics \& Child Health. 19(6). pp. 305-309.

4. Sainsbury, K., Mullan, B., Sharpe, L. (2013) A randomized controlled trial of an online intervention to improve gluten-free diet adherence in celiac disease. American Journal of Gastroenterology. 108(5). pp. 811-817.

5. Samasca, G., Sur, G., Lupan, I., Deleanu, D. (2014) Gluten-free diet and quality of life in celiac disease. Gastroenterology and Hepatology from Bed to Bench. 7(3). pp. 139-143.

6. Zingone, F., Swift, G.L., Card, T.R., Sanders, D.S., Ludvigsson, J.F., Bai, J.C. (2015) Psychological morbidity of celiac disease: A review of the literature. United European Gastroenterology Journal. 3(2). pp. 136-145.

7. Ludvigsson, J.F., Card, T., Ciclitira, P.J., Swift, G.L., Nasr, I., Sanders, D.S., et al. (2015) Support for patients with celiac disease: A literature review. United European Gastroenterology Journal. 3(2). pp. 146-159.

8. Häuser, W., Stallmach, A., Caspary, W., Stein, J. (2006) Predictors of reduced health-related quality of life in adults with coeliac disease. Alimentary Pharmacology \& Therapeutics. 25(5). pp. 569-578.

9. De Rosa, A., Troncone, A., Vacca, M., Ciacci, C. (2004) Characteristics and quality of illness behavior in celiac disease. Psychosomatics. 45(4). pp. 336-342.

10. Nachman, F., del Campo, MP, González, A., Corzo, L., Vázquez, H., Sfoggia, C., et al. (2010) Long-term deterioration of quality of life in adult patients with celiac disease is associated with treatment noncompliance. Digestive and Liver Disease. 42(10). pp. 685-691.

11. Barratt, S.M., Leeds, J.S., Sanders, D.S. (2011) Quality of life in coeliac disease is determined by perceived degree of difficulty adhering to a gluten-free diet, not the level of dietary adherence ultimately achieved. Journal of Gastrointestinal and Liver Diseases. 20(3). pp. 241245.

12. Hopman, E., Koopman, H., Wit, J., Mearin, M. (2009) Dietary compliance and health-related quality of life in patients with coeliac disease. European Journal of Gastroenterology \& Hepatology. 21(9). pp. 1056-1061.

13. Grudeva, L. (2018) Optimizirane na klinichniya podhod i dietolechenieto pri glutenova enteropatiya. Varna: Gea Print. pp.173-177. (in Bulgarian). 